\title{
miR-200b suppresses cell proliferation, migration and enhances chemosensitivity in prostate cancer by regulating Bmi-1
}

\author{
JUNJIE YU, YOUYI LU, DI CUI, ENHUI LI, YIPIN ZHU, YUYANG ZHAO, FUJUN ZHAO and SHUJIE XIA \\ Department of Urology, Shanghai First People's Hospital, School of Medicine, \\ Shanghai Jiaotong University, Shanghai 200080, P.R. China
}

Received October 25, 2013; Accepted November 18, 2013

DOI: $10.3892 /$ or.2013.2897

\begin{abstract}
RNAs (miRNAs) are a class of small noncoding RNAs that can post-transcriptionally regulate gene expression and play critical roles in many important biological processes. The role of miRNAs in prostate cancer (PCa) development and pathogenesis remains largely unknown. In the present study, we showed that miR-200b was downregulated in clinical prostatic tumors when compared to normal prostate tissue and in advanced PCa cell lines when compared to normal epithelial prostatic cells. Enforced miR-200b expression suppressed PCa cell proliferation and migration and enhanced chemosensitivity to docetaxel by targeting B-cell-specific Moloney murine leukemia virus insertion site 1 (Bmi-1). Bmi-1 was detected at higher levels in $\mathrm{PCa}$, and knockdown of Bmi-1 showed similar effects as miR-200b overexpression in PCa cells. Moreover, we confirmed that these effects were correlated with increased levels of E-cadherin and P16 and a reduction in vimentin expression and expression of stem cell markers (CD44 and OCT4). These findings suggest that miR$200 \mathrm{~b}$ plays vital roles as a tumor-suppressor by targeting Bmi-1 and may be a promising therapeutic target for PCa treatment.
\end{abstract}

\section{Introduction}

Prostate cancer (PCa) is one of the most common lethal malignant diseases in males in the United States (1). Disease confined to the prostate is treatable, while metastatic PCa eventually leads to accelerated disease progression and mortality (2). Thus, to further improve patient survival, it is essential to further understand the molecular and cellular mechanisms of PCa development and to establish novel therapeutic strategies to target PCa progression and metastasis.

Correspondence to: Professor Shujie Xia or Dr Fujun Zhao Department of Urology, Shanghai First People's Hospital, School of Medicine, Shanghai Jiaotong University, 100 Haining Road, Hongkou, Shanghai 200080, P.R. China

E-mail: xsjurologist@163.com

E-mail: zhaofujun72@163.com

Key words: miR-200b, prostate cancer, Bmi-1, proliferation, migration
microRNAs (miRNAs) are a class of highly conserved, small non-coding, single-stranded RNAs, 17-25 nucleotides in length, that regulate gene expression via post-transcriptional inhibition of protein synthesis (3). These small RNAs negatively regulate approximately one-third of human coding genes by binding to complementary sites in the 3'-untranslated region (3'-UTR) of their target genes (4). miRNAs may also play critical roles in the pathogenesis of PCa. It is anticipated that identification and characterization of PCa-related miRNAs may shed light on understanding the molecular mechanisms of disease development and are therefore crucial for the development of novel therapies.

The miR-200 family (including miR-200a, miR-200b, miR-200c and miR-141) is a cluster of miRNAs highly correlated with epithelial-mesenchymal transition (EMT), wherein miR-200b was identified as a critical regulator of tumor invasion, metastasis and chemosensitivity (5). Increasing evidence suggests that miR-200b acts as a tumor-suppressive miRNA and is a critical regulator of EMT, cancer stem cell (CSC) maintenance, apoptosis and cancer chemosensitivity $(6,7)$. However, the functional role and mechanism of involvement of miR-200b during the development and pathogenesis of $\mathrm{PCa}$ remain to be determined.

B-cell-specific Moloney murine leukemia virus insertion site $1(\mathrm{Bmi}-1)$ is a member of the polycomb $(\mathrm{PcG})$ family of epigenetic gene silencers. Bmi-1 is frequently overexpressed in several types of human cancers and may play an important role in carcinogenesis (8-11). Ectopic expression of Bmi-1 can directly promote EMT and malignancy in human nasopharyngeal epithelial cells, whereas silencing endogenous Bmi-1 expression can reverse EMT (12). Bmi-1 also plays a critical role in the maintenance of stem cells (13) and is a crucial regulator of self-renewal in adult prostate cells (14). However, the regulatory mechanism of Bmi-1 and its role in PCa cells require further research.

In the present study, we hypothesized that miR-200b may influence the biological behaviors of PCa cells by inhibiting the expression of Bmi-1. Our results revealed that upregulation of miR-200b effectively downregulated the expression of Bmi-1 and also inhibited cell proliferation and cell migration and promoted chemosensitivity. These findings provide evidence that miR-200b acts as a tumor-suppressor gene and suggest that miR-200b may be a potential new strategy to prevent prostate progression by targeting the oncogene Bmi-1. 
Moreover, we demonstrated that silencing of Bmi-1 revealed its function as an oncogene in $\mathrm{PCa}$.

\section{Materials and methods}

Tissue specimens. Human PCa specimens were obtained from 30 patients who underwent radical prostatectomies at the Department of Urology, Shanghai First People's Hospital, School of Medicine, Shanghai Jiaotong University between March 2011 and December 2012. Fifteen benign prostatic hyperplasia (BPH) tissue samples used as the control were obtained by transurethral resection of the prostate (TURP). All of the samples were confirmed by pathological examination and stored in liquid nitrogen for miRNA analysis. Formalinfixed, paraffin-embedded samples for immunohistochemistry from $60 \mathrm{PCa}$ tissues and $30 \mathrm{BPH}$ tissues obtained by radical prostatectomy and TURP, respectively, were from the Tissue Paraffin Block Bank of Pathology in our hospital. The Institutional Review Board of Shanghai First People's Hospital approved all experimental procedures, and patient consent was obtained before tissue collection.

Cell lines and cell culture. Human PCa cell lines LNCaP, PC3 and DU145 were obtained from the Shanghai Cell Bank, the Chinese Academy of Sciences, and the benign hyperplastic epithelial cell line BPH-1 was developed in our laboratory. All cell lines were maintained in RPMI-1640 medium (Gibco-BRL, Rockville, MD, USA) supplemented with $50 \mathrm{U} / \mathrm{ml}$ penicillin, $50 \mathrm{mg} / \mathrm{ml}$ streptomycin and $10 \%$ fetal bovine serum (Gibco-BRL) in a humidified atmosphere at $37^{\circ} \mathrm{C}$ in $5 \% \mathrm{CO}_{2}$.

Real-time quantitative RT-PCR. Total RNA from PCa cell lines was extracted using TRIzol reagent (Invitrogen Life Technologies, Carlsbad, CA, USA). Real-time quantitative RT-PCR was carried out using the PrimeScript Reverse Transcription System and SYBR Premix Ex Taq ${ }^{\mathrm{TM}}$ II kit (Takara, Dalian, China) according to the manufacturer's instructions. The primer sequences used were as follows: Bmi-1 (forward primer, 5'-TGGACTGACAAATGCTGGAG-3' and reverse primer, 5'-GGCAAACAAGAAGAGGTGGA-3'); internal control GAPDH (forward primer, 5'-TCGACAGTCA GCCGCATCTTCTTT-3' and reverse primer, 5'-ACCAAATC CGTTGACTCCGACCTT-3'). PCR conditions included denaturation at $94^{\circ} \mathrm{C}$ for $2 \mathrm{~min}$, followed by 40 cycles of $5 \mathrm{sec}$ at $94^{\circ} \mathrm{C}, 30 \mathrm{sec}$ at $60^{\circ} \mathrm{C}$ and $30 \mathrm{sec}$ at $72^{\circ} \mathrm{C}$.

Expression of mature miR-200b was assayed using stem-loop RT followed by real-time PCR analysis. BulgeLoop $^{\text {TM }}$ miRNA qRT-PCR Primer Set and miRNA qRT-PCR Control Primer Set (RiboBio, Guangdong, China) were used for quantitative real-time PCR analysis of miR-200b and U6 small nuclear RNA, respectively. PCR conditions included denaturation at $95^{\circ} \mathrm{C}$ for $20 \mathrm{sec}$, followed by 40 cycles of $10 \mathrm{sec}$ at $95^{\circ} \mathrm{C}, 20 \mathrm{sec}$ at $60^{\circ} \mathrm{C}$ and $10 \mathrm{sec}$ at $70^{\circ} \mathrm{C}$. U6 expression was used as an internal control for miR-200b expression. The relative expression fold change of miRNAs and mRNAs was calculated using the $2^{-\Delta \Delta C t}$ method (15). All experiments were performed in triplicates.

Transient transfections. PC3 and DU145 cells seeded at $1.5 \times 10^{5}$ cells/well in 6 -well plates were transfected with
miR-200b mimic and a scramble control (RiboBio) performed at a concentration of $50 \mathrm{nM}$ using Lipofectamine 2000 reagent (Invitrogen Life Technologies) according to the manufacturer's instructions. Transfection efficiency was confirmed by qRT-PCR. For the silencing experiments, PC3 and DU145 cells were transfected with small interfering RNAs (siRNAs) targeting Bmi-1 (siRNA-Bmi-1) and their negative controls (siRNA-NC) (RiboBio) using Lipofectamine 2000 reagent according to the manufacturer's instruction. The sequences of the siRNA were identical to those described in a previous study (16). Forty-eight hours after transfection, cell proliferation and migration were assayed.

Cell Counting Kit-8 (CCK-8) and EdU analysis. Cell proliferation was assessed using the CCK-8 assay (Dojindo, Kumamoto, Japan). The transfected PC3 and DU145 cells were plated with $100 \mu \mathrm{l}$ culture mediun in 96-well plates at a density of 3,000 cells/well. After 24, 48 and $72 \mathrm{~h}, 10 \mu \mathrm{l}$ of CCK-8 reagent $(5 \mathrm{mg} / \mathrm{ml})$ was added to each well, and incubation was carried out at $37{ }^{\circ} \mathrm{C}$ for $2 \mathrm{~h}$. Viable cells were evaluated by absorbance measurements at $450 \mathrm{~nm}$. Each assay was performed in 6 replicates for 3 independent experiments. The impact of miR-200b on PC3 and DU145 cell proliferation was also assessed by the EdU cell proliferation assay kit, according to the manufacturer's instructions. The assays were performed as recommended by the manufacturer of the EdU detection kit Apollo 488 (RiboBio).

Migration assay. The migratory capacity of the PCa cells following miR-200b and siRNA-Bmi-1 or NC transfection was assessed using a Corning Transwell assay according to the manufacturer's protocol. A total of $0.5 \times 10^{5} \mathrm{PCa}$ cells in $200 \mu \mathrm{l}$ of serum-free medium were seeded into the upper chamber of the system. Lower chambers were filled with $0.75 \mathrm{ml}$ of complete medium. After $24 \mathrm{~h}$ of incubation, the cells in the upper chamber were removed with a cotton swab, and the transmigrated cells were fixed in methanol and stained with crystal violet. Stained cells were counted by photographing 5 fields/membrane.

Chemosensitivity assay. Rates of sensitivity to drugs were determined by the cell proliferation reagent CCK-8. Forty-eight hours after transfection, cells were digested and plated in 96-well plates at a density of 3,000 cells/well. After an overnight incubation, the cells were treated with docetaxel $(10 \mathrm{nM})$. After $72 \mathrm{~h}, 10 \mu \mathrm{l}$ of CCK-8 reagent was added to each well and the plate was incubated at $37^{\circ} \mathrm{C}$ for $2 \mathrm{~h}$. Viable cells were evaluated by absorbance measurements at $450 \mathrm{~nm}$. Each assay was performed in 6 replicates in 3 independent experiments.

Immunofluorescence staining. BPH-1, LNCaP, PC3 and DU145 cells were grown on glass coverslips, fixed with $4 \%$ paraformaldehyde, permeabilized with $0.5 \%$ Triton X-100 and then blocked with $5 \%$ bovine serum albumin in phosphatebuffered saline (PBS). The coverslips were then exposed to the primary antibody, anti-Bmi-1, overnight at $4^{\circ} \mathrm{C}$, followed by secondary antibodies. Images were captured using a laser scanning confocal fluorescence microscope (LSM-510; Carl Zeiss, Jena, Germany). 
A

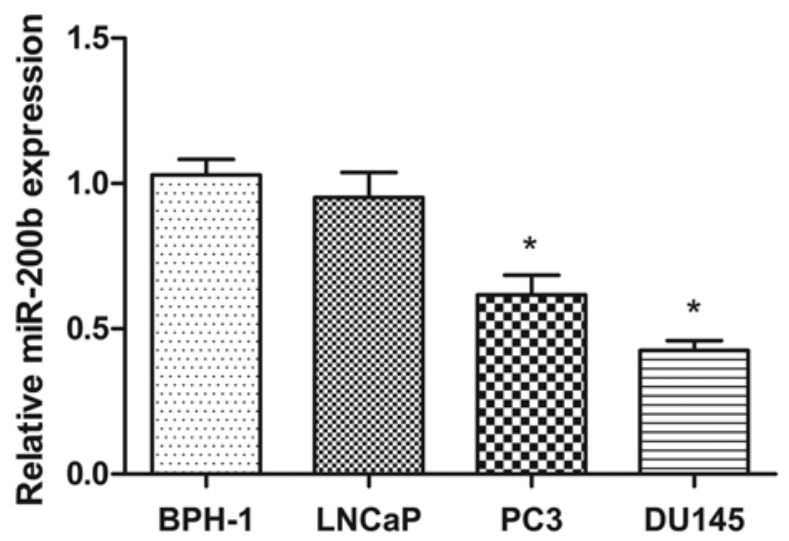

B

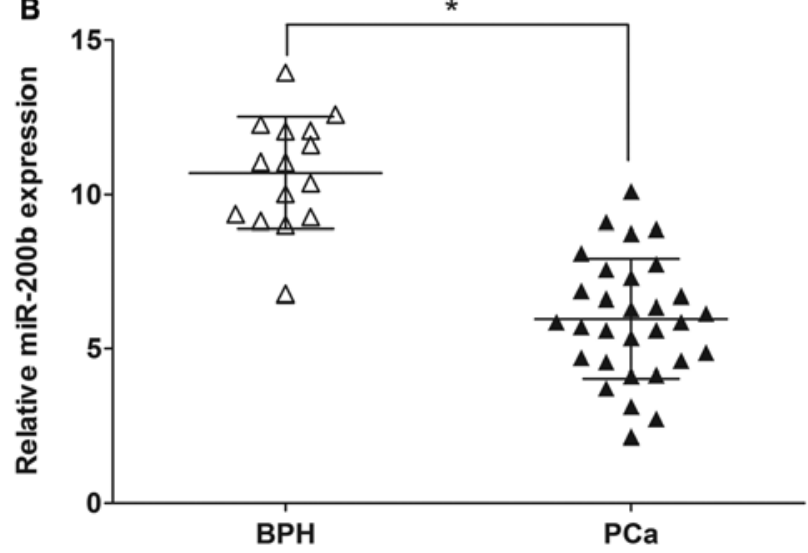

Figure 1. Expression of miR-200b is downregulated in prostate cancer. (A) qRT-PCR confirmation of the relative miR-200b expression levels in a normal prostate epithelial cell line (BPH-1) and 3 PCa cell lines (LNCaP, PC3 and DU145). ${ }^{*} \mathrm{P}<0.05$, when compared with the BPH-1 cell line. (B) miR-200b expression was determined in $30 \mathrm{PCa}$ tissues and $15 \mathrm{BPH}$ non-tumor tissues by qRT-PCR. Data were normalized to the RNA control U6 snRNA. * $<0.05$, when compared with the $\mathrm{BPH}$ tissues. $\mathrm{BPH}$, benign prostatic hyperplasia; $\mathrm{PCa}$, prostate cancer.

Western blot analysis. Cells were washed twice with cold PBS and homogenized in ice-cold RIPA buffer (Beyotime, Jiangsu, China) containing phosphatase and protease inhibitors. Total protein was separated by denaturing SDS-polyacrylamide gel electrophoresis and transferred electrophoretically onto PVDF membranes. Membranes were incubated with the primary antibodies for rabbit anti-Bmi-1, rabbit anti-E-cadherin, vimentin, P16 and rabbit anti-GAPDH (Cell Signaling Technology, Inc., Beverly, MA, USA) at $4^{\circ} \mathrm{C}$ overnight and subsequently with horseradish peroxidase-conjugated secondary antibody. Signals were visualized with an ECL chemiluminescence kit (Boster, Wuhan, China) and exposed to X-ray film.

Immunohistochemical staining of tissues. Immunohistochemistry was conducted on archived paraffin-embedded formalin-fixed tissues of the study patients. Bmi-1 immunohistochemistry was performed according to the avidin-biotin-peroxidase complex method, with primary antibodies specific against human Bmi-1 (Cell Signaling Technology, Inc.) used at a 1:400 dilution. The secondary antibody (Long Island Biotech, Shanghai, China) was applied at a dilution of 1:400. Bmi-1 immunoreactivity was assessed by the intensity of the positive reaction (no staining, 0 ; low staining, 1; medium staining, 2; strong staining, 3). Immunohistochemical evaluation was performed in a blinded manner by two independent pathologists.

Statistical analyses. Statistical analyses were performed using the Statistical Package for the Social Sciences software version 17.0 (SPSS, Inc., Chicago, IL, USA). Data are presented as means \pm SD from at least 3 independent experiments. The two-tailed $t$-test and $\chi^{2}$ tests were used to assess statistically significant differences. A P-value $<0.05$ was considered to indicate a statistically significant result.

\section{Results}

miR-200b is reduced in PCa when compared with normal prostate cells and tissues. We determined the expression levels of miR-200b in human prostate cell lines, which included prostate carcinoma cell lines (LNCaP, PC3 and DU145) and the benign hyperplastic epithelial cell line BPH-1 by real-time qPCR analysis. We found that miR-200b was expressed at significantly lower levels in the PC3 and DU145 cells when compared with that in the non-malignant BPH-1 cells, while there was no difference between the BPH-1 and LNCaP cells (Fig. 1A). We next investigated the clinical relevance of miR-200b. Its expression levels were analyzed by real-time qPCR in PCa tissue and BPH tissue samples. miR$200 \mathrm{~b}$ expression was significantly downregulated in the cancer tissues when compared with that in the BPH tissue samples $(\mathrm{P}<0.05)$ (Fig. 1B)

miR-200b suppresses cell proliferation, migration and improves chemosensitivity to docetaxel. We performed gain-of-function studies using miR-200b mimics, and miRNA transfection efficiency was determined by real-time qRT-PCR. After PC3 and DU145 cells were transfection with $50 \mathrm{nM}$ miR-200b mimics and negative control, the expression of miR-200b was significantly upregulated (Fig. 2A). The effects of ectopic expression of miR-200b on cell proliferation in PC3 and DU145 cells were examined by CCK-8 assay. Transfection with miR-200b mimics resulted in a significant decrease in cell growth of the PCa cell lines $(\mathrm{P}<0.05)$ (Fig. 2B). We further investigated the effect of $\mathrm{miR}-200 \mathrm{~b}$ on cell proliferation using an EdU incorporation assay. Fewer EdU-positive cells were observed in the miR-200b mimic-transfected cells when compared to the NC-transfected cells (Fig. 2C). These data indicate that miR-200b has a vital role in reducing the growth of PCa cells.

A Transwell assay was introduced to investigate whether miR-200b regulates tumor migration. PC3 and DU145 cells were transfected with either miR-200b mimics or the negative control. PCa cells transfected with miR-200b exhibited a decrease in cell motility (Fig. 2D). Our results are consistent with a previous study that miR-200b inhibits EMT and cancer cell migration (17). Moreover, miR-200b-treated PC3 and DU145 cells showed higher chemosensitivity to $10 \mathrm{nM}$ 
A

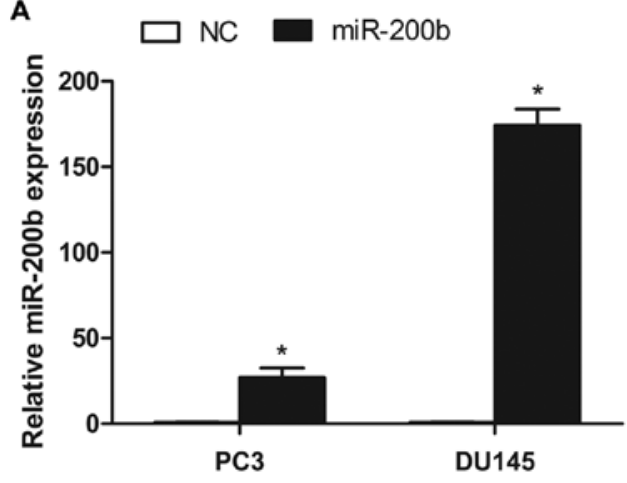

B
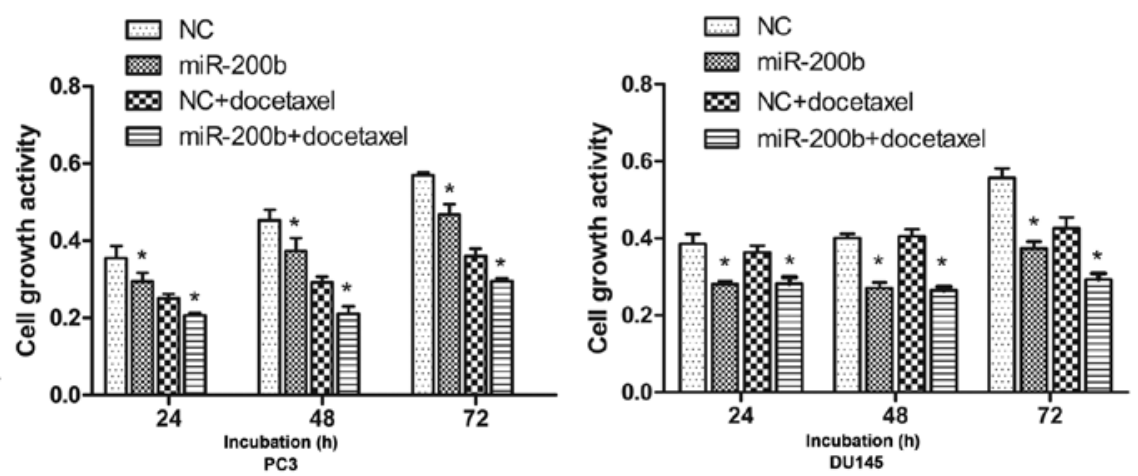

C
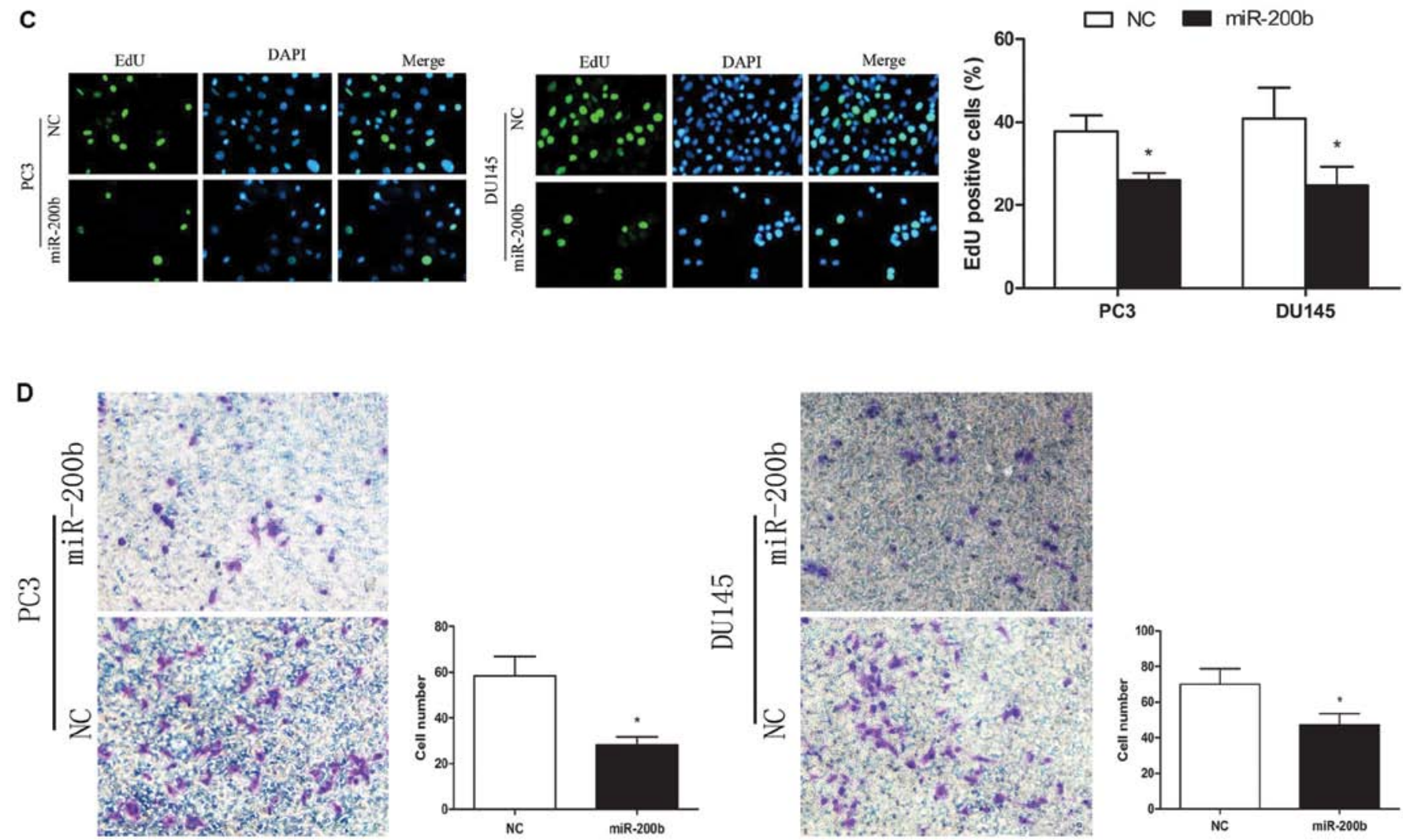

Figure 2. Upregulation of miR-200b inhibits proliferation, migration and enhanced sensitivity to docetaxel. (A) Forty-eight hours after transfection, miR-200b expression was enhanced when compared with that in the corresponding negative control group in PC 3 and DU145 cell lines. (B) Cellular proliferation was measured in the control and miR-200b mimic-transfected PC3 and DU145 cells using an CCK-8 assay at 3 time points. CCK-8 assay of miR-200b- and control-transfected PC3 and DU145 cells following exposure to $10 \mathrm{nM}$ docetaxel. Each sample was tested in triplicate. (C) Representative micrographs (left panel) and quantification of EdU incorporated-cells after transfection with miR-200b mimics or control. The percentage of positive cells was derived from triplicate samples (original magnification, x400). (D) PC3 and DU145 cells that were transfected with miR-200b showed less migratory potential than that of the control miRNA-transfected cells. Cells were incubated in Transwell chambers, and the migrated cells at the bottom of the insert were stained and counted after $24 \mathrm{~h}$. Representative images are shown at the left. Histograms (right panel) represent the median of 3 independent experiments (original magnification, $\mathrm{x} 200)$. ${ }^{*} \mathrm{P}<0.05$ when compared with the control group. CCK-8, Cell Counting Kit-8.

docetaxel than the NC-treated cells (Fig. 2B), demonstrating that upregulation of miR-200b improved PC3 and DU145 cell chemosensitivity to docetaxel.

miR-200b inhibits Bmi-1 and increases E-cadherin expression in PCa cells. Real-time qRT-PCR demonstrated that Bmi-1 mRNA expression was decreased in PC3 and DU145 cells after transfection with miR-200b mimics when compared with the negative control (Fig. 3A). Furthermore, the protein expression level of Bmi-1 was also markedly reduced in the
PCa cell lines after miR-200b transfection (Fig. 3B). These results indicate that Bmi-1 may be a target of miR-200b in PC3 and DU145 cells. Furthermore, enforced expression of miR200b in PC 3 and DU145 cells resulted in increased expression of E-cadherin and reduced the expression of vimentin when compared with the control cells (Fig. 3C).

Bmi-1 is efficiently inhibited by siRNA. To explore the biological functions of Bmi-1 in PC3 and DU145 cells, we transfected these two cell lines with siRNA-Bmi-1. The 
A
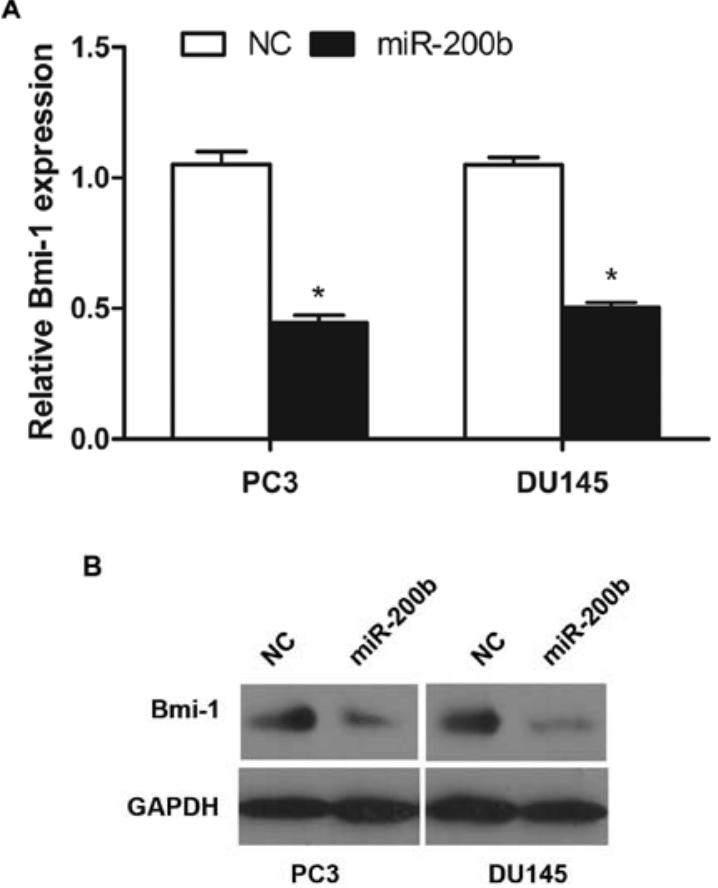

C

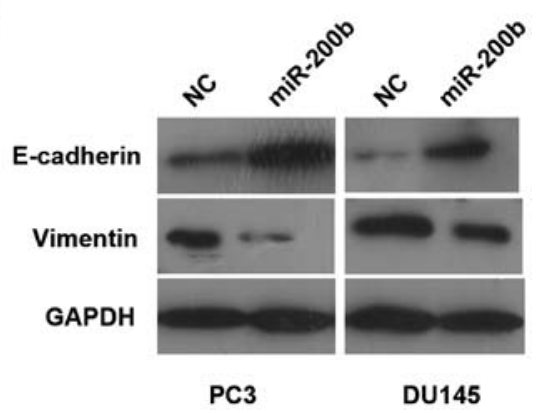

Figure 3. Effects of miR-200b on the expression of Bmi-1 in PC3 and DU145 PCa cells. (A) The expression of Bmi-1 mRNA was evaluated by real-time qPCR after transfection of miR-200b for $48 \mathrm{~h}$. The Bmi-1 mRNA expression level was reduced in the miR-200b-transfected PCa cells when compared with the level in the control cells. GAPDH was used as an internal control ${ }^{*} \mathrm{P}<0.05$. (B) Western blotting showed that the Bmi-1 protein level was decreased in the miR-200b-transfected PC3 and DU145 cells. (C) E-cadherin was increased while the vimentin protein level was decreased in the PC3 and DU145 cells following transfection. The results were normalized to GAPDH expression. Bmi-1, B-cell-specific Moloney murine leukemia virus insertion site 1.

efficiency of siRNA-Bmi-1 inhibition was measured by qRT-PCR and western blotting. The Bmi-1 mRNA (Fig. 4A) and protein (Fig. 4D) levels were downregulated in the PC3 and DU145 cells following transfection with Bmi-1 siRNA. These data showed that the expression of Bmi-1 was efficiently silenced by Bmi-1 siRNA in the PCa cells. We selected the Bmi-1 siRNA to further study the functional consequences of downregulation of Bmi-1 expression on cell growth and migration.

Bmi-1 modulates PCa cell proliferation, motility and chemosensitivity. Transfection of PC3 and DU145 cells with siRNA-Bmi-1 significantly decreased cell proliferation when compared with the corresponding negative controls (Fig. 4B). Since previous studies have shown that ectopic Bmi-1 suppresses the expression of tumor-suppressor p16 (18), in the present study the p16 protein level was determined by western blotting. Downregulation of Bmi-1 expression by siRNA-Bmi-1 transfection significantly upregulated p16 protein expression (Fig. 4D). The Transwell chamber migration assay revealed that the motility of DU145 and PC3 cells was dramatically hampered by the ablation of Bmi-1 (Fig. 4C). Knockdown of Bmi-1 protein led to the upregulation of E-cadherin and downregulation of vimentin at the protein levels (Fig. 4D). Moreover, siRNA-Bmi-1-treated PCa cells showed higher chemosensitivity to $10 \mathrm{nM}$ docetaxel when compared to the NC-treated cells (Fig. 4B), suggesting that Bmi-1 silencing enhances docetaxel activity. Previous studies have shown that Bmi-1 knockdown decreased expression of stem cell-like markers and chemoresistance markers (19). In the present study, we also found that the mRNA expression levels of stemness genes CD44 and OCT4 were downregulated in the siRNA-Bmi-1-treated cells when compared with the control siRNA cells (Fig. 4E). These results suggest that the suppression of Bmi-1 decreases cell proliferation and motility and improves chemosensitivity to docetaxel.

Bmi-1 is overexpressed in PCa. To investigate differential expression between prostate cancer cell lines and the normal prostate cells, we measured the Bmi-1 protein level in 3 different human PCa cell lines (LNCaP, PC3 and DU145) and the benign hyperplastic epithelial cell line BPH-1 using western blot assay (Fig. 5A). Immunofluorescence staining also showed that expression of Bmi-1 was higher in the $\mathrm{PCa}$ cell lines than that in the BPH-1 cells (Fig. 5B). We then examined Bmi-1 expression in 30 cases of BPH and 60 malignant prostate tissue samples. The staining intensity was generally increased in the malignant cells. The Bmi-1 antibody resulted in a strong, predominantly nuclear staining in the malignant epithelia, whereas normal epithelial cells showed significantly lower staining intensities when compared with the $\mathrm{PCa}$ tissue $\left(\mathrm{P}<0.05, \chi^{2}\right.$ tests) (Fig. $\left.5 \mathrm{C}\right)$. These data showed that Bmi-1 plays a vital role as an oncogene in vitro and in vivo.

\section{Discussion}

miRNAs have recently been described as important players in human cancer and their role as therapeutic targets has been proposed. The expression of miRNAs was found to be markedly deregulated in prostate cancer (PCa), strongly suggesting that miRNAs are involved in the initiation and progression of this disease (20). Recent reports indicate that miR-200b is highly correlated with epithelial-mesenchymal transition (EMT) and is identified as a critical regulator of tumor invasion, metastasis and chemosensitivity $(6,21)$. The miR-200 family was found to directly target the mRNA of the E-cadherin transcriptional repressors ZEB1 and ZEB2. Ectopic expression of miR-200 reduced cancer cell motility by upregulation of E-cadherin protein levels. Conversely, inhibition of miR-200 reduced E-cadherin expression, increased expression of vimentin and induced EMT (22). Moreover, recent studies have discovered that miR-200b and miR-1 inhibit EMT via a Slug-dependent mechanism and tumorigenesis via a Slug-independent mechanism (23). In the present study, we confirmed that miR-200b expression levels 
A

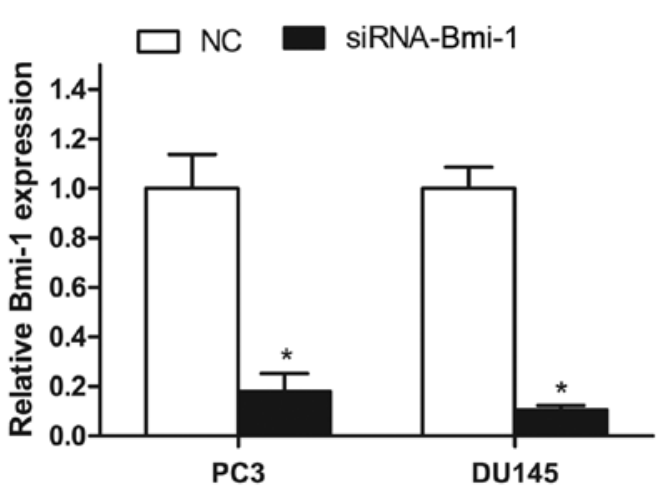

C
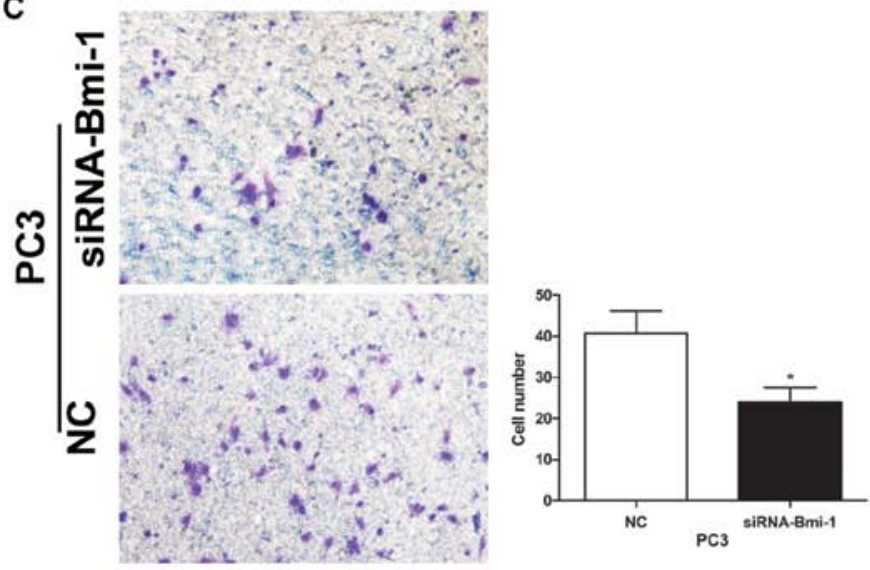

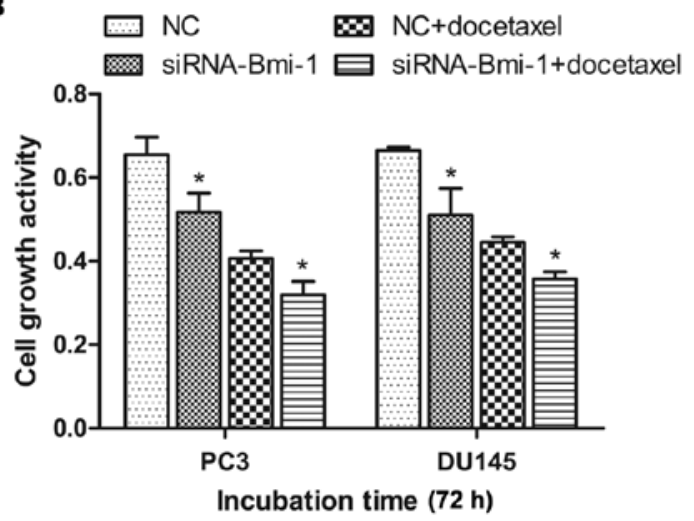

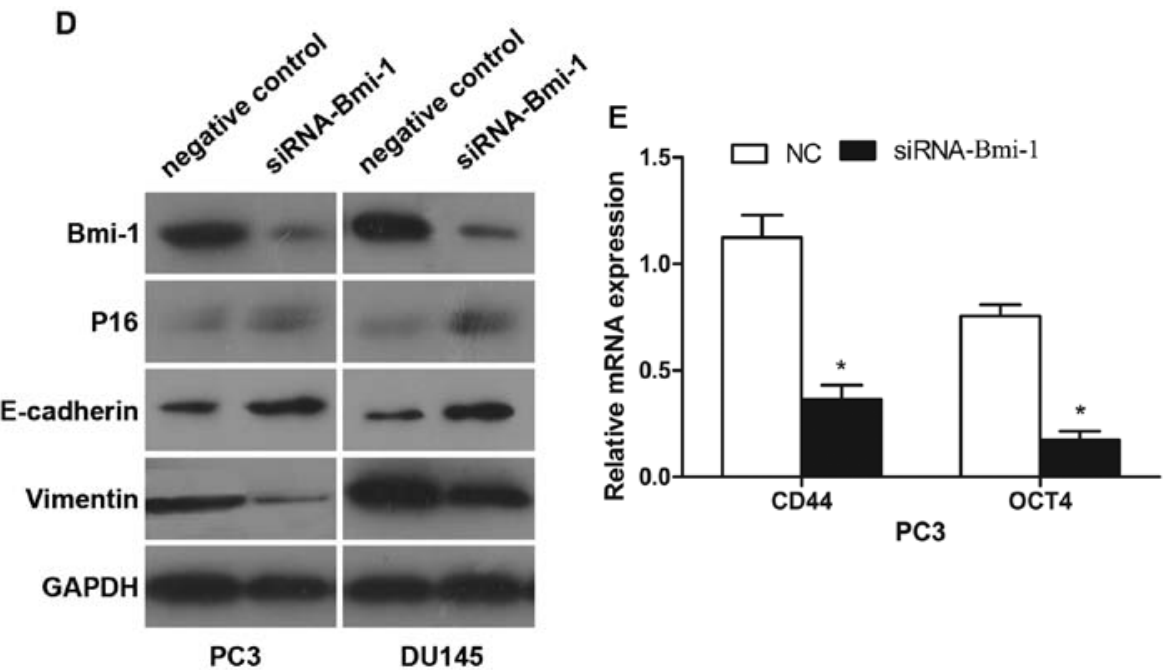
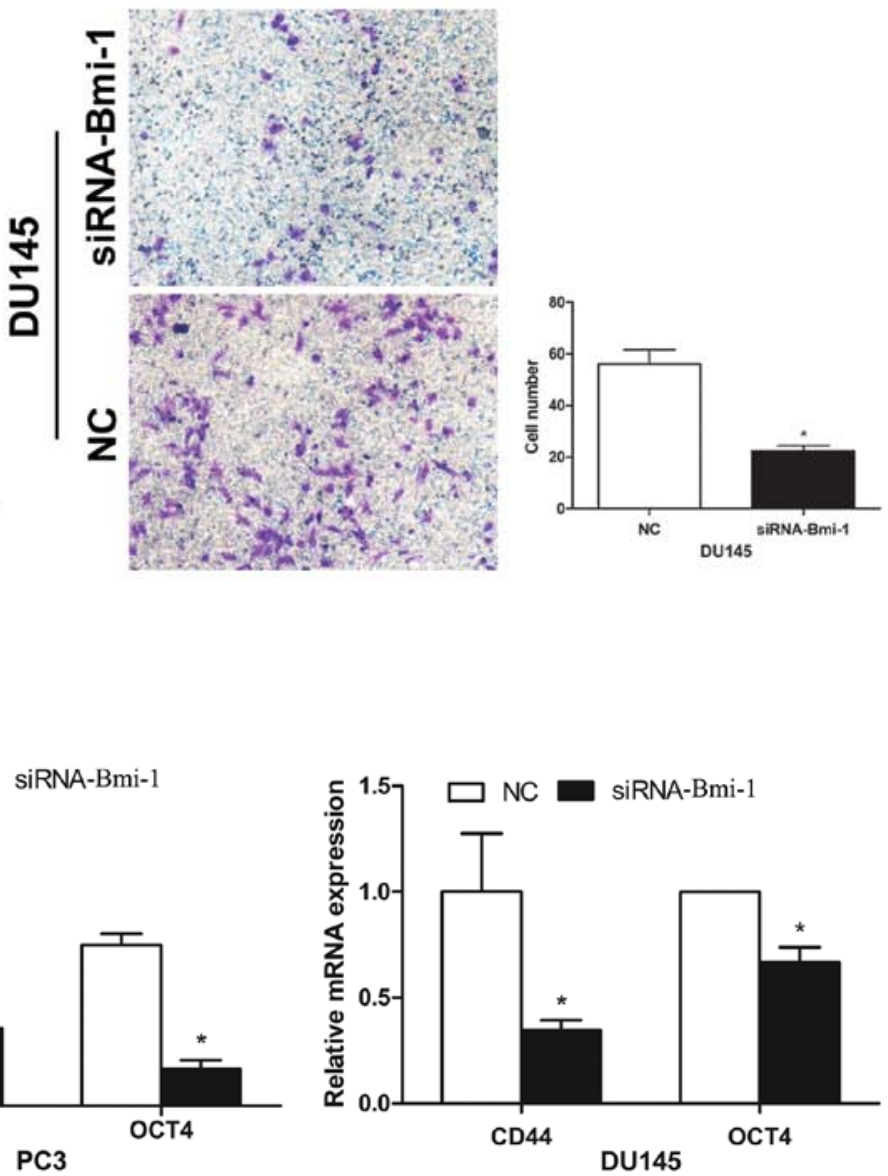

Figure 4. Inhibition of Bmi-1 by siRNAs in PCa cells. Silencing of Bmi-1 inhibits PCa cell proliferation, motility and promotes chemosensitivity. (A) Bmi-1 expression was confirmed by qRT-PCR and was dramatically decreased by RNA interference in PC3 and DU145 cells. (B) Bmi-1 knockdown inhibited cell growth and enhanced sensitivity to $10 \mathrm{nM}$ docetaxel as compared with the $\mathrm{NC}$ at $72 \mathrm{~h}$ post-transfection. (C) The migratory capacity was analyzed using the Transwell chamber assay (original magnification, x200). (D) Western blot analysis of the protein expression of Bmi-1, p16, E-cadherin, vimentin in siRNA-Bmi-1 and control cells, respectively. (E) mRNA expression levels of CD44 and OCT4 in the siRNA-Bmi-1- and control siRNA-transfected PC3 and DU145 cells were determined by qRT-PCR. "P $<0.05$ when compared with the control group. Bmi-1, B-cell-specific Moloney murine leukemia virus insertion site 1; siRNAs, small interfering RNAs; PCa, prostate cancer.

were underexpressed in advanced prostate cancer cell lines and in PCa clinical specimens. Consistent with our results, miR-200b has frequently been found to be downregulated in cancers, including gastric (24), lung cancer (25), renal cell carcinoma (26) and bladder cancer (27), as well as PCa (28).
The downregulation of miR-200b in PCa indicates that it may play a vital role in PCa tumor development and progression as a potential tumor-suppressor.

In PCa cells, miR-200b has an impact on pathways governing cell proliferation, drug sensitivity and cell migration. We found 
A

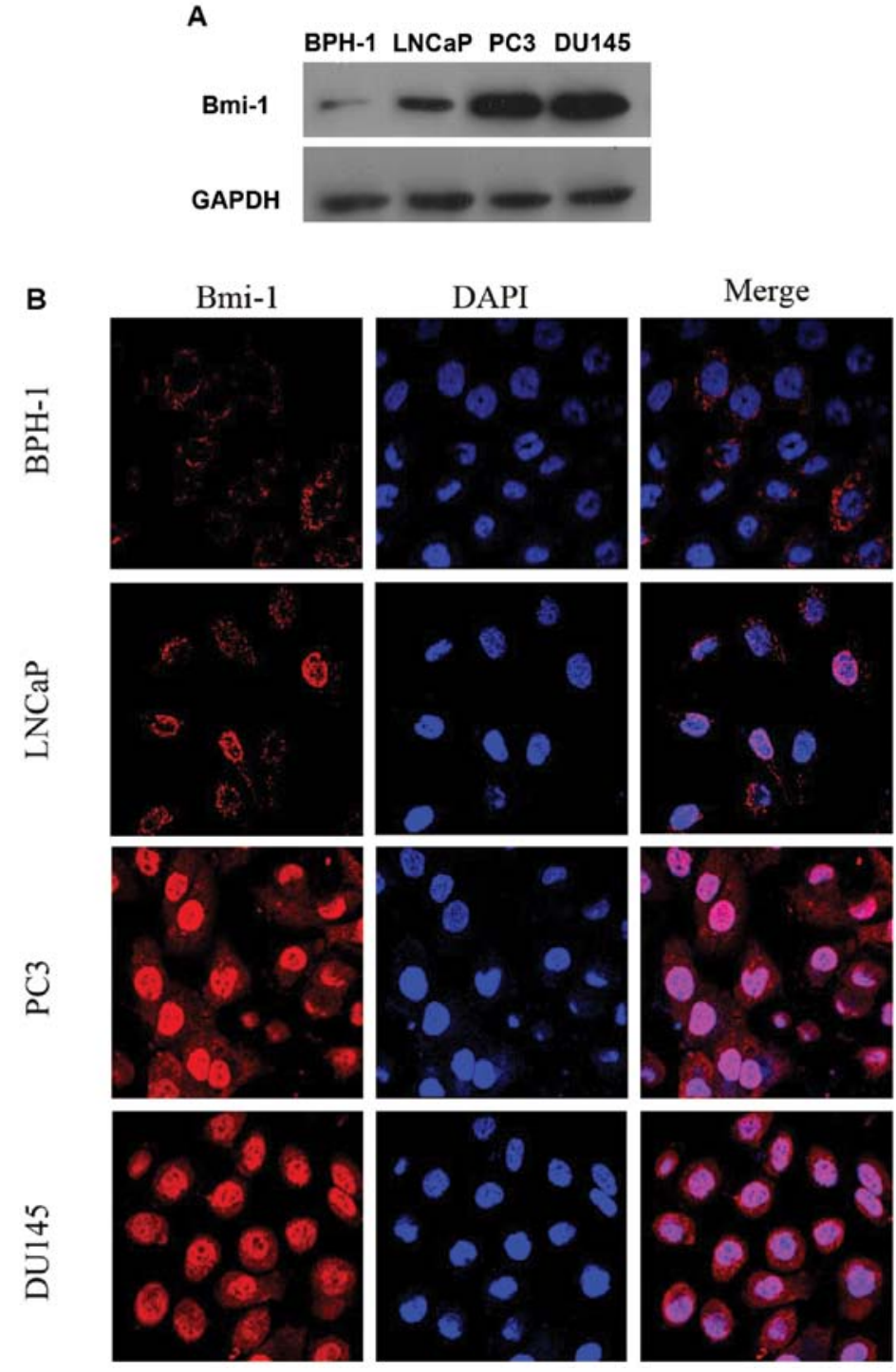

C a

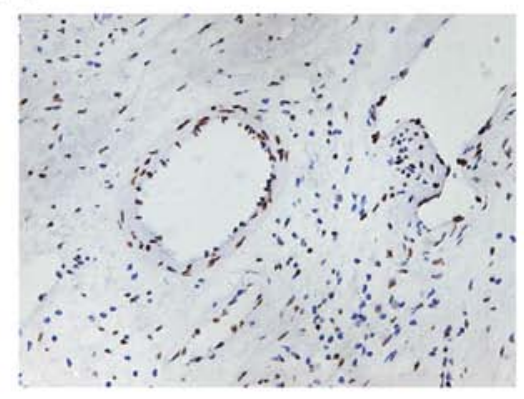

b

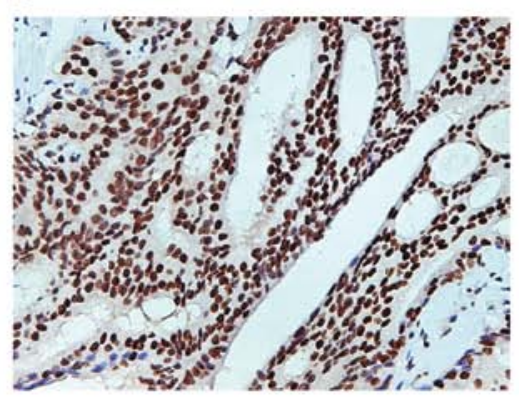

c

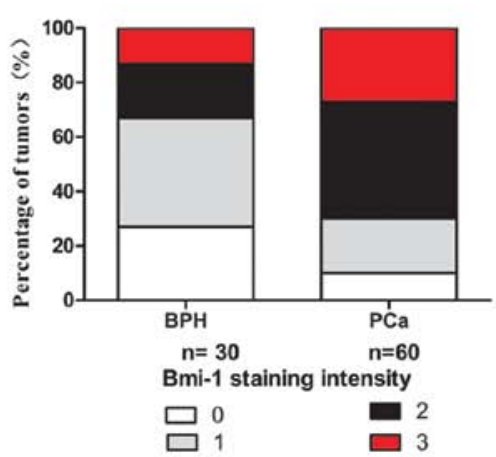

Figure 5. Expression of Bmi-1 in prostate cancer cell lines and patient tissue samples. (A) The expression of Bmi-1 protein levels in BPH-1, LNCaP, PC3 and DU145 cell lines. GAPDH was used as an internal control. (B) Immunofluorescence staining in human prostate adenocarcinoma cell lines LNCaP, PC3 and DU145 and human prostate epithelium cell line BPH-1 showed that Bmi-1 was overexpressed in different degrees in the prostate cancer cell lines, when compared with the BPH-1 cell line. (C) Different expression levels of Bmi-1 in normal prostate and prostate cancer tissues. a, Nonmalignant epithelia showing low staining; b, malignant cells showing strong staining. c, Scoring of the staining intensities with the anti-Bmi-1 antibody indicates that Bmi-1 protein expression was significantly increased in PCa when compared with the BPH tissue specimens (original magnification, $\mathrm{x} 400)$. $\mathrm{P}<0.05$ ( $\chi^{2}$ tests). Bmi-1, B-cell-specific Moloney murine leukemia virus insertion site 1; BPH, benign prostatic hyperplasia.

that overexpression of miR-200b in PCa cells suppressed cell growth and cell migration. Moreover, PC3 and DU145 cells showed a higher sensitivity to $10 \mathrm{nM}$ docetaxel when cells were treated with miR-200b mimics, when compared to the negative control. These findings demonstrate that miR-200b may have significant future clinical implications. 
In addition, we further explored the mechanism by which miR-200b affects proliferation, migration and sensitivity to docetaxel in PCa cells. We used target prediction databases (miRanda, Targetscan and PicTar) to search for genes regulated by miR-200b and found that miR-200b may target Bmi-1. Recent research has revealed that miR-200b functions as a tumor-suppressor gene and that Bmi-1 may be the target of miR-200b in human tongue cancer (29).

Previous studies have suggested that Bmi-1 acts as an oncogene and plays a crucial role in cell proliferation, transformation, EMT, self-renewal of stem cells and cancer initiation and chemoresistance in various human tumors $(19,30,31)$. We discovered that Bmi-1 was overexpressed in PCa tumor tissues when compared with that in the BPH normal tissues. Bmi-1 overexpression was also observed in PCa cell lines when compared with the BPH-1 normal prostate epithelium cells. Consistent with our findings, previous publications also reported that Bmi-1 was overexpressed in $\mathrm{PCa}(32,33)$, and its overexpression was associated with a high Gleason score and increased risk of recurrence after prostatectomy (34). In the present study, we showed that rescue of miR-200b expression led to downregulation of Bmi-1 mRNA and protein levels in PCa cells. These results suggest that miR-200b may directly target the 3'-UTR of Bmi-1 mRNA and act as a potential tumor-suppressor in $\mathrm{PCa}$.

We demonstrated that knockdown of Bmi-1 in PC3 and DU145 cells significantly reduced cell proliferation and upregulated the p16 tumor-suppressor. There is an enormous body of evidence suggesting that Bmi-1 regulates cell proliferation and senescence through suppressing p16 (35). Previous studies have discovered that targeting Bmi-1 improves the outcome of docetaxel therapy in animal models bearing chemoresistant prostatic tumors (32). Crea et al (36) identified that Bmi-1 silencing impairs antioxidant defense and sensitizes PCa cells to docetaxel. The present study also found that inhibition of Bmi-1 enhanced the antitumor activity of docetaxel in $\mathrm{PCa}$ cells. These results indicate that Bmi-1 may be exploited as a potential molecular target for therapeutics to treat chemoresistant tumors. Recent studies have demonstrated that many metastatic cancers undergo EMT resulting in enhanced cell motility and invasion (37). The hallmark of EMT is the loss of adherens protein E-cadherin and acquisition of mesenchymal markers such as vimentin (38). A recent study showed that Bmi-1 is regulated by Twist1 in HNSCC and is essential in EMT (39). Yet, the role of Bmi-1 in PCa cell migration has not been thoroughly explored. The present study is consistent with the literature reported. Following inhibition of the expression of Bmi-1, the migration ability of PCa cells was reduced. Here we also provided evidence that Bmi-1 is correlated with E-cadherin and vimentin protein expression in PCa cells.

In summary, our data suggest that Bmi-1 is expressed at a high level in PCa. miR-200b plays a pivotal role in $\mathrm{PCa}$ at least in part via downregulation of the oncogene Bmi-1 and may serve as a therapeutic target by which to inhibit PCa cell proliferation and migration and enhance chemosensitivity.

\section{Acknowledgements}

The present study was supported by a grant from the National Natural Science Foundation of China (no. 81072114).

\section{References}

1. Siegel R, Ward E, Brawley O and Jemal A: Cancer statistics, 2011: the impact of eliminating socioeconomic and racial disparities on premature cancer deaths. CA Cancer J Clin 61: 212-236, 2011.

2. Damber JE and Aus G: Prostate cancer. Lancet 371: 1710-1721, 2008.

3. Bartel DP: MicroRNAs: genomics, biogenesis, mechanism, and function. Cell 116: 281-297, 2004.

4. Meister G, Landthaler M, Patkaniowska A, Dorsett Y, Teng G and Tuschl T: Human Argonaute2 mediates RNA cleavage targeted by miRNAs and siRNAs. Mol Cell 15: 185-197, 2004.

5. Feng B, Wang R and Chen LB: Review of miR-200b and cancer chemosensitivity. Biomed Pharmacother 66: 397-402, 2012.

6. Zhu W, Xu H, Zhu D, et al: miR-200bc/429 cluster modulates multidrug resistance of human cancer cell lines by targeting BCL2 and XIAP. Cancer Chemother Pharmacol 69: 723-731, 2012.

7. Wellner U, Schubert J, Burk UC, et al: The EMT-activator ZEB1 promotes tumorigenicity by repressing stemness-inhibiting microRNAs. Nat Cell Biol 11: 1487-1495, 2009.

8. Guo BH, Feng Y, Zhang R, et al: Bmi-1 promotes invasion and metastasis, and its elevated expression is correlated with an advanced stage of breast cancer. Mol Cancer 10: 10, 2011.

9. Bhattacharya R, Nicoloso M, Arvizo R, et al: MiR-15a and MiR-16 control Bmi-1 expression in ovarian cancer. Cancer Res 69: 9090-9095, 2009.

10. Song $\mathrm{W}$, Tao $\mathrm{K}, \mathrm{Li} \mathrm{H}$, et al: Bmi-1 is related to proliferation, survival and poor prognosis in pancreatic cancer. Cancer Sci 101: 1754-1760, 2010.

11. He X, Dong Y, Wu CW, et al: MicroRNA-218 inhibits cell cycle progression and promotes apoptosis in colon cancer by downregulating BMI1 polycomb ring finger oncogene. Mol Med 18: 1491-1498, 2013.

12. Song LB, Li J, Liao WT, et al: The polycomb group protein Bmi-1 represses the tumor-suppressor PTEN and induces epithelialmesenchymal transition in human nasopharyngeal epithelial cells. J Clin Invest 119: 3626-3636, 2009.

13. Molofsky AV, Pardal R, Iwashita T, Park IK, Clarke MF and Morrison SJ: Bmi-1 dependence distinguishes neural stem cell self-renewal from progenitor proliferation. Nature 425: 962-967, 2003.

14. Lukacs RU, Memarzadeh $\mathrm{S}, \mathrm{Wu} \mathrm{H}$ and Witte $\mathrm{ON}$ : Bmi-1 is a crucial regulator of prostate stem cell self-renewal and malignant transformation. Cell Stem Cell 7: 682-693, 2010.

15. Livak KJ and Schmittgen TD: Analysis of relative gene expression data using real-time quantitative PCR and the $2^{-\Delta \Delta \mathrm{C}_{\mathrm{T}}}$ method. Methods 25: 402-408, 2001.

16. Onder TT, Kara N, Cherry A, et al: Chromatin-modifying enzymes as modulators of reprogramming. Nature 483: 598-602, 2012.

17. Korpal M, Lee ES, Hu G and Kang Y: The miR-200 family inhibits epithelial-mesenchymal transition and cancer cell migration by direct targeting of E-cadherin transcriptional repressors ZEB1 and ZEB2. J Biol Chem 283: 14910-14914, 2008.

18. Fan C, He L, Kapoor A, et al: Bmil promotes prostate tumorigenesis via inhibiting $\mathrm{p} 16^{\mathrm{INKAA}}$ and $\mathrm{p} 14^{\mathrm{ARF}}$ expression. Biochim Biophys Acta 1782: 642-648, 2008.

19. Dong P, Kaneuchi M, Watari H, et al: MicroRNA-194 inhibits epithelial to mesenchymal transition of endometrial cancer cells by targeting oncogene BMI-1. Mol Cancer 10: 99, 2011.

20. Schaefer A, Jung M, Mollenkopf HJ, et al: Diagnostic and prognostic implications of microRNA profiling in prostate carcinoma. Int J Cancer 126: 1166-1176, 2010.

21. Kong D, Banerjee S, Ahmad A, et al: Epithelial to mesenchymal transition is mechanistically linked with stem cell signatures in prostate cancer cells. PLoS One 5: e12445, 2010.

22. Park SM, Gaur AB, Lengyel E and Peter ME: The miR-200 family determines the epithelial phenotype of cancer cells by targeting the E-cadherin repressors ZEB1 and ZEB2. Genes Dev 22: 894-907, 2008.

23. Liu YN, Yin JJ, Abou-Kheir W, et al: MiR-1 and miR-200 inhibit EMT via Slug-dependent and tumorigenesis via Slugindependent mechanisms. Oncogene 32: 296-306, 2013.

24. Tang H, Kong Y, Guo J, et al: Diallyl disulfide suppresses proliferation and induces apoptosis in human gastric cancer through Wnt-1 signaling pathway by up-regulation of miR-200b and miR-22. Cancer Lett 340: 72-81, 2013. 
25. Pacurari M,Addison JB,Bondalapati N, et al: The microRNA-200 family targets multiple non-small cell lung cancer prognostic markers in H1299 cells and BEAS-2B cells. Int J Oncol 43: 548-560, 2013

26. Yoshino H, Enokida H, Itesako T, et al: Epithelial-mesenchymal transition-related microRNA-200s regulate molecular targets and pathways in renal cell carcinoma. J Hum Genet 58: 508-516, 2013.

27. Köhler CU, Bryk O, Meier S, et al: Analyses in human urothelial cells identify methylation of miR-152, miR-200b and miR-10a genes as candidate bladder cancer biomarkers. Biochem Biophys Res Commun 438: 48-53, 2013.

28. Cao Q, Mani RS, Ateeq B, et al: Coordinated regulation of polycomb group complexes through microRNAs in cancer. Cancer Cell 20: 187-199, 2011

29. Sun L, Yao Y, Liu B, et al: MiR-200b and miR-15b regulate chemotherapy-induced epithelial-mesenchymal transition in human tongue cancer cells by targeting BMI1. Oncogene 31: 432-445, 2012

30. Kang MK, Kim RH, Kim SJ, et al: Elevated Bmi-1 expression is associated with dysplastic cell transformation during oral carcinogenesis and is required for cancer cell replication and survival. Br J Cancer 96: 126-133, 2007.

31. Siddique HR and Saleem M: Role of BMI1, a stem cell factor, in cancer recurrence and chemoresistance: preclinical and clinical evidences. Stem Cells 30: 372-378, 2012.

32. Siddique HR, Parray A, Tarapore RS, et al: BMI1 polycomb group protein acts as a master switch for growth and death of tumor cells: regulates TCF4-transcriptional factor-induced BCL2 signaling. PLoS One 8: e60664, 2013.
33. Wolters T, Vissers KJ, Bangma $\mathrm{CH}$, Schröder $\mathrm{FH}$ and van Leenders GJ: The value of EZH2, p27 ${ }^{\text {kipl }}$, BMI-1 and MIB-1 on biopsy specimens with low-risk prostate cancer in selecting men with significant prostate cancer at prostatectomy. BJU Int 106: 280-286, 2010.

34. van Leenders GJ, Dukers D, Hessels D, et al: Polycomb-group oncogenes EZH2, BMI1, and RING1 are overexpressed in prostate cancer with adverse pathologic and clinical features. Eur Urol 52: 455-463, 2007.

35. Jacobs JJ, Kieboom K, Marino S, DePinho RA and van Lohuizen M: The oncogene and Polycomb-group gene bmi-1 regulates cell proliferation and senescence through the ink $4 a$ locus. Nature 397: 164-168, 1999.

36. Crea F, Duhagon Serrat MA, Hurt EM, Thomas SB, Danesi R and Farrar WL: BMI1 silencing enhances docetaxel activity and impairs antioxidant response in prostate cancer. Int J Cancer 128: 1946-1954, 2011.

37. Thiery JP, Acloque H, Huang RY and Nieto MA: Epithelialmesenchymal transitions in development and disease. Cell 139: 871-890, 2009

38. Sánchez-Tilló E, Lázaro A, Torrent R, et al: ZEB1 represses E-cadherin and induces an EMT by recruiting the SWI/SNF chromatin-remodeling protein BRG1. Oncogene 29: 3490-3500, 2010.

39. Yang MH, Hsu DS, Wang HW, et al: Bmi1 is essential in Twist1induced epithelial-mesenchymal transition. Nat Cell Biol 12: 982-992, 2010. 